\title{
Ensayo clínico controlado y aleatorizado para determinar los efectos del uso de Pelotas de Parto durante el trabajo de parto: Estudio piloto.
}

\section{AUTORES:}

Beatriz Elena Delgado-García. DUE. Máster en Ciencias de la Enfermería. Especialista en Enfermería Obstétrico-Ginecológica. Hospital VInalopó Salud.

Mª Isabel Orts-Cortés. DUE. Máster en Ciencias de la Enfermería. Dpto. de Enfermería. Escuela Universitaria de Enfermería. Universidad de Alicante.

Alberto Poveda-Bernabeu. Diplomado en Enfermería. Especialista en Enfermería Obstétrico-Ginecológica. Hospital General Universitario de Alicante.

Pablo Caballero-Pérez. Licenciado en Ciencias Estadísticas. Profesor asociado. Dpto. Enfermería Comunitaria, Med. Prev. y Salud Pública e Ha Ciencia. EUE. Universidad Alicante.

\section{INSTITUCIÓN DONDE SE REALIZÓ EL ESTUDIO:}

Hospital General Universitario de Alicante, perteneciente al Departamento 19 de Salud de la Comunidad Valenciana. (C/ Pintor Baeza s/n 03010 - Alicante, España)

\section{AGRADECIMIENTOS:}

A las mujeres que han participado en nuestro estudio, al personal de paritorio del H. G. U. Alicante y a la Unidad Docente de Matronas de la Escuela Valenciana de Estudios de la Salud.

El proyecto de investigación del este estudió fue presentado y defendido en formato póster en el XI Encuentro Internacional de Investigación en Enfermería celebrado en Madrid (España) en Noviembre 2007 con el título "Esferodinamia durante el trabajo de parto" y obteniendo el Premio al mejor póster del "Rincón de Residentes". 
El estudio, ya concluido, fue aceptado y presentado como comunicación en el XIII Congreso Nacional de Matronas celebrado en Alicante (España) en Octubre 2008; así como en el XII Encuentro Internacional de Investigación en Enfermería y 6 Coloquio Bianual Joanna Briggs celebrado en Córdoba (España) en Noviembre 2008, con el título "Estudio experimental sobre la eficacia de la esferodinamia durante el trabajo de parto".

\section{RESUMEN}

OBJETIVO. Determinar los efectos usar la Pelota de Parto durante el trabajo de parto en relación al tiempo de dilatación y expulsivo, la integridad perineal, la percepción de la intensidad del dolor y la seguridad.

MÉTODO. Ensayo clínico controlado y aleatorizado. Participantes: 18 a 35 años, nulíparas, bajo riesgo, a término. Intervención: Realización de movimientos sentadas sobre PdP durante el parto. Variables resultado: Tiempo de dilatación y expulsivo; integridad perineal; percepción del dolor, recuerdo del dolor en el puerperio y pre-post intervención; tipo de parto; motivo de distocia; Apgar; ingreso en UCI neonatal. Análisis: comparación de grupos: t-Student para variables continuas y Ji-cuadrado para categóricas. Significación si p<0.05.

RESULTADOS: 58 participantes (3 pérdidas). El tiempo de dilatación y expulsivo, y la integridad perineal fue similar entre grupos. A los $4 \mathrm{~cm}$. el grupo experimental refirió menos dolor que el grupo control; 6,9 puntos vs 8,2. La diferencia en la percepción del dolor recordada en el puerperio inmediato fue de 1,48 puntos, mayor en el grupo control. La medición del dolor en el grupo experimental antes del uso de la PG fue de 7,45 puntos y tras la intervención de 6,07 puntos. Los tres resultados resultaron estadísticamente significativos En las variables relacionadas con la seguridad no hubo diferencias entre los grupos.

CONCLUSIÓN: El uso de Pelotas de Parto disminuye la percepción del dolor de parto y es segura. 


\section{ABSTRACT}

OBJECTIVE. To determine the effects using the birth ball during obstetric labor on first and second labor stage, perineal integrity, perception of pain intensity and safety.

METHOD. Randomized controlled trial. Participants: 18 to 35 years, nulliparous, low-risk, at term. Intervention: Making movements sitting on BB during obstetric labor. Main outcomes: Duration of first and second labor stages; perineal integrity; pain perception, his memory in the postpartum period and pre-post intervention, type of delivery, cause of dystocia, Apgar, neonatal ICU admission. Analysis: Comparison of groups: t-Student for continuous variables and chi-square test for categorical. Significance if $p<0.05$.

RESULTS: 58 participants (3 losses). Time of first and second stage, and perineal integrity ware similar between groups. At $4 \mathrm{~cm}$. experimental group referred less pain than control group, 6.9 points vs 8.2. Difference in the memory of pain in the immediate postpartum period was 1.48 points higher in control group. The measurement of pain in experimental group before the use of the BB was of 7.45 points, and after the intervention of 6.07 points. These three results were statistically significant. In safety-related variables there were no differences between groups.

CONCLUSION: The use of Birth Ball decreases obstetric labor pain perception and it is safe. 


\section{¿QUÉ SE CONOCE?}

Realizar ejercicios moderado y masaje perineal durante el embarazo, y durante la dilatación permitir libertad de movimientos preferiblemente en posición vertical, optimiza el proceso de parto. El uso de la Pelota de Parto reúne éstas recomendaciones, por lo que cabría esperar esos mismos efectos beneficiosos.

\section{¿QUÉ APORTA?}

El uso de la Pelota de Parto durante el trabajo de parto disminuye la percepción de dolor. No se han observado efectos no deseados maternos ni neonatales.

\section{INTRODUCCIÓN}

Actualmente los modelos de asistencia al parto están siendo objeto de revisión, realizándose investigaciones para mejorar la calidad asistencial basándose en la evidencia científica, como respuesta a la demanda social y de los profesionales, apoyados por las administraciones públicas ${ }^{1}$.

La evidencia científica recomienda ejercicios aeróbicos durante el embarazo ${ }^{2}$ y entrenar el suelo pélvico pues optimiza el proceso de parto y disminuye la incontinencia urinaria del puerperio ${ }^{3}$. Masajear el periné durante el embarazo previene el trauma perineal y disminuye el dolor postparto ${ }^{4}$. La libertad de movimientos y las posiciones verticales durante el parto acortan el periodo de dilatación, disminuyen del dolor y el trauma perineal, mejoran la satisfacción materna y los resultados neonatales ${ }^{5}$.

En los últimos años se han introducido prácticas nuevas como el uso de Pelotas de Parto ${ }^{1}$ (PdP) con las gestantes. Las PdP son pelotas de goma, resistentes y capaces de sostentar el peso de un adulto sentado encima.

Con una intervención se realizan varias de las recomendaciones comentadas: usar la PdP conlleva estar en posición vertical, realizando movimientos que movilizan los músculos y articulaciones de la pelvis, masajeando el periné, y posibilitando el afrontamiento del dolor 
de manera activa.

Tras revisar la literatura, no hemos encontrado estudios experimentales sobre la PdP que avalen su seguridad ni eficacia, aunque existen monografías ${ }^{6}$ y artículos descriptivos $^{7-11}$. Las PdP aparecen citadas en diferentes revisiones ${ }^{1,12}$ como posible método no farmacológico de manejo del dolor de parto, pero pendiente de investigación.

El objetivo es determinar los efectos del uso de PdP durante el trabajo de parto en relación al tiempo de dilatación y expulsivo, la integridad perineal y la percepción de la intensidad del dolor, así como su seguridad con respecto a la salud de la madre y el feto, a través de un ensayo clínico controlado y aleatorizado.

\section{METODOS}

\section{Diseño y ámbito.}

Ensayo clínico controlado y aleatorizado. Servicio de partos del Hospital General Universitario de Alicante. Departamento 19, Comunidad Valenciana.

\section{Participantes.}

Criterios de inclusión: gestantes, 18 a 35 años, nulíparas, a término, feto único, cefálica, gestación controlada, con consentimiento informado firmado.

Criterios de exclusión: alteraciones del equilibrio o limitación física, problemas de comunicación, antecedentes obstétricos, problemas en la gestación actual o enfermedad materna que conlleve morbilidad asociada.

\section{Selección y distribución de la muestra.}

Muestreo no probabilístico consecutivo desde de Septiembre 2007 a Febrero 2008. Asignación aleatoria simple.

\section{Variables}

La PdP es un balón hinchable de goma ( 65 o 75cm. de diámetro). Intervención: la gestante se sienta sobre la pelota y realiza movimientos en posición vertical de balanceo y rotación de la pelvis. Tiempo mínimo establecido: 20 minutos. Las rodillas quedan más bajas que la pelvis, los pies apoyados en el suelo, las manos libres para un apoyo extra ${ }^{10}$ estando la 
mujer estable ${ }^{17}$ y siempre acompañada. Entre la pelota y la mujer se sitúa un empapador por higiene. Además tras cada uso las pelotas son limpiadas ${ }^{12}$. La intervención era interrumpida en caso de prescripción facultativa, problemas para lograr un RCG adecuado, alteraciones en el patrón FCF o indisposición de la gestante.

El grupo control tuvo libertad de movimientos y el resto de cuidados semejantes al grupo experimental, salvo ofrecerle la PdP. Los investigadores clínicos fueron entrenados para proporcionar las mismas indicaciones.

Resultados principales: Tiempo de dilatación, tiempo de expulsivo, grado de integridad perineal y percepción de la intensidad del dolor (Escala Analógica Visual) registrada a los 4 $\mathrm{cm}$. de dilatación y en el puerperio inmediato (dolor global del proceso).

En el grupo experimental el dolor fue medido también pre y post intervención (tras 20 min. de uso).

La seguridad de la intervención se midió por el tipo de finalización de parto, motivo de distocia, test de APGAR y la necesidad de ingreso en UCI neonatal.

Otras variables: sociodemográficas y de control (al inicio, durante y al finalizar el parto).

Procedimiento y recogida de datos.

Verificados los criterios de selección, se solicitó el consentimiento informado. Recogida de datos a través de la historia clínica y la entrevista personal. Para verificar la metodología se realizó una prueba piloto de Junio a Agosto de 2007, tras la aprobación del protocolo del estudio por el comité de ética e investigación del Hospital y respetando los principios éticos de la declaración de Helsinki.

\section{Análisis de datos.}

Análisis descriptivo de las variables categóricas (frecuencias y proporciones) y continuas (media, desviación estándar). Evaluación de la comparabilidad inicial de los grupos, durante la dilatación y el puerperio. Contraste de hipótesis bilateral con test paramétricos (t-Student) para variables continuas y test no paramétricos (Ji-cuadrado) para variables categóricas. Nivel de significación $\mathrm{p}<0.05$. Datos analizados con Statistical Package for the Social Sciences (SPSS) para Windows v. 16. 


\section{RESULTADOS}

Total de 58 participantes: 34 del grupo experimental (pérdidas: 2 por tiempo insuficiente de intervención y 1 por no desear realizarla) y 24 del grupo control.

Las características sociodemográficas, las condiciones de inicio y las otras variables de control fueron similares en ambos grupos (tabla 1).

El tiempo de dilatación medio de 2 a 4cm. fue de 250,50 min. (DE $\pm 143,9)$ para el grupo experimental y para el control $267,4 \mathrm{~min}$. (DE $\pm 140,9$ ); de $4 \mathrm{~cm}$. a completa fue de $229,4 \mathrm{~min}$. $(\mathrm{DE} \pm 116,7)$ para el grupo experimental y para el control $248,1 \mathrm{~min}$. (DE $\pm 175,5)$; el tiempo medio de expulsivo fue de $93,2 \mathrm{~min}$. $(\mathrm{DE} \pm 79,8)$ para el grupo experimental y para el control 78,5min. (DE $\pm 54,4)$; sin diferencias estadísticamente significativas.

A los $4 \mathrm{~cm}$. el grupo experimental refirió menos dolor que el grupo control; 6,9 puntos vs 8,2. La diferencia en la percepción del dolor recordada en el puerperio inmediato fue de 1,48 puntos, mayor en el grupo control. Ambos resultados estadísticamente significativos (tabla 2).

El dolor en el grupo experimental $(n=30)$ antes del uso de la PdP fue de 7,45 puntos $(\mathrm{DE} \pm 2,12)$ y después de 6,07 puntos $(\mathrm{DE} \pm 2,11)$, diferencia estadísticamente significativa (diferencia de medias=1,38; $I C_{95}$ de la diferencia=0,81-1,96;t=4,098; $g l=29 ; p<0,001$ ) (figura 1).

En el grupo experimental hubo un mayor porcentaje de mujeres con periné integro, un $33,3 \%(n=7)$ frente a un $9,1 \%(n=2)$ del grupo control; el porcentaje de episiotomías fue superior en el grupo control, $81,8 \%(n=18)$ frente al $66,7 \% \quad(n=14)$ (figura 2$)$. No hubo desgarros de III ni IV grado. El análisis se realizó con dos categorías: periné íntegro y no íntegro (desgarros de II grado y episiotomías). Las diferencias entre grupos resultaron no estadísticamente significativas ( $\mathrm{Ji}$-cuadrado $=3,81 ; g \mathrm{l}=1 ; \mathrm{p}=0.051$ ).

En las variables relativas a la seguridad de la intervención no se encontraron diferencias estadísticamente significativas entre los grupos. Ningún recién nacido requirió hospitalización en la UCI neonatal. 


\section{DISCUSIÓN}

No hemos encontrado estudios experimentales en los que se evaluara el uso de la PdP para poder comparar nuestros resultados. Por ello éste trabajo es pionero en un campo en el que, nos consta que el uso de la PdP se está generalizando'.

De los resultados destaca que usar la PdP diminuye significativamente la percepción del dolor, así como su recuerdo en el puerperio inmediato, en comparación con el grupo control, y en el grupo experimental se obtuvo una disminución estadísticamente significativa en la percepción del dolor pre y post intervención.

Las estrategias de afrontamiento activo que aumentan la sensación de control están relacionadas con una disminución del dolor y aumento de satisfacción materna ${ }^{13}$. Por tanto, el uso de la PdP podría utilizarse como método alternativo no farmacológico del manejo del dolor del parto.

El masaje perineal durante el parto ha sido evaluado en distintos estudios en los cuales no se han observado beneficios en la integridad perineal ${ }^{14}$. En este estudio el grado de integridad perineal es similar entre los dos grupos de comparación, aunque los datos descriptivos muestran que el grupo control presenta un mayor porcentaje de perinés no íntegros.

Las diferencias entre grupos encontradas en los tiempos medios de la dilatación y el expulsivo no han resultado estadísticamente significativas. Durante el estudio no se observó ningún efecto adverso materno ni neonatal.

Una de las limitaciones del estudio es el tamaño muestral; entre las causas sucedió que las mujeres que habían realizado la intervención comentaron favorablemente su experiencia a otras mujeres que al llegar nos solicitaban usar la PDP, siendo excluídas por su predisposición hacía la intervención. Otros factores externos impidieron la recogida de algunos datos, como pe.: analgesia epidural antes de los $4 \mathrm{~cm}$ de dilatación, ingreso con más de $4 \mathrm{~cm}$ de dilatación,.. que han hecho que en algunos análisis bivariados la frecuencia recogida sea inferior al tamaño muestral. 
Dada la naturaleza de la intervención no fue posible realizar ninguna técnica de enmascaramiento, si bien existió el compromiso de que la única diferencia en el trato a los grupos fuese el uso de la PdP. El análisis de los datos fué de cegado.

Nuestra muestra, reducida para elevar sus resultados a definitivos, debe considerarse como un estudio piloto que marca un camino a seguir; son necesarios nuevos estudios y muestras más numerosas para que los resultados que se obtengan, respalden las hipótesis de partida.

No hemos evaluado objetivamente el efecto de la intervención sobre la percepción de la mujer en términos de satisfacción, aunque fue muy bien acogida por la población y de manera informal se recogieron comentarios como "sentirse más cómoda y activa". También los profesionales acogieron bien la PdP, integrandola en su práctica habitual. Consideramos necesario que en futuros estudios se evalúen estos aspectos.

\section{CONCLUSIONES}

Los resultados muestran que el uso de PdP disminuye la percepción del dolor, sin efectos negativos sobre los resultados maternos ni neonatales.

Es necesario investigar sobre éstas y otras variantes del empleo de la PDP con mayores tamaños muestrales para llegar a conclusiones definitivas y poder extrapolar los resultados. 


\section{$\underline{\text { TABLAS }}$}

Tabla 1. Características previas y otras variables de control

\begin{tabular}{|c|c|c|c|}
\hline \multirow[t]{2}{*}{ Características } & \multicolumn{2}{|c|}{ Grupo } & \multirow[t]{2}{*}{ Valor $p$} \\
\hline & $\begin{array}{l}\text { Experimental } \\
\quad(n=31)\end{array}$ & $\begin{array}{l}\text { Control } \\
(n=24)\end{array}$ & \\
\hline Edad (años, media [DE]) & $29,00(4,61)$ & $27,75(5,78)$ & 0,39 \\
\hline Edad gestacional (semanas, media [DE]) & $39,81(1,37)$ & $39,88(0,89)$ & 0,84 \\
\hline Ganancia ponderal (kg, media [DE]) & $14,26(4,50)$ & $15,44(8,70)$ & 0,64 \\
\hline Educación maternal (número; sí/ no) & $23 / 8$ & $12 / 12$ & 0,06 \\
\hline $\begin{array}{l}\text { Forma de inicio de parto (número; } \\
\text { espontáneo/ inducción o estimulación) }\end{array}$ & $14 / 17$ & $10 / 14$ & 0,80 \\
\hline Líquido amniótico (número; claro/ teñido) & $29 / 2$ & $22 / 2$ & 0.79 \\
\hline $\begin{array}{l}\text { Rotura prematura de membranas (número; } \\
\text { si /no) }\end{array}$ & $10 / 21$ & $7 / 17$ & 0.81 \\
\hline $\begin{array}{l}\text { Test de Bishop (número; cérvix } \\
\text { inmaduro/ intermedio/ maduro) }\end{array}$ & $5 / 13 / 13$ & $4 / 12 / 8$ & 0.79 \\
\hline Ritmo máximo de oxitocina (ml/h, media [DE]) & $\begin{array}{r}26,37(18,83) \\
n=30\end{array}$ & $\begin{array}{r}22,25(13,03) \\
n=24\end{array}$ & 0,35 \\
\hline $\begin{array}{l}\text { Tiempo de permanencia de oxitocina (horas, } \\
\text { media [DE]) }\end{array}$ & $\begin{array}{r}7,5(4,27) \\
\mathrm{n}=30\end{array}$ & $\begin{array}{r}6,8(4,11) \\
n=24\end{array}$ & 0,54 \\
\hline $\begin{array}{l}\text { Momento solicitud de analgesia (cm. dilatación, } \\
\text { media }[D E] \text { ) }\end{array}$ & $\begin{array}{r}4,26(2,09) \\
\mathrm{n}=23\end{array}$ & $\begin{array}{r}3,73(1,24) \\
n=22\end{array}$ & 0,32 \\
\hline Administración de analgesia (número; sí/no) & $24 / 7$ & $19 / 5$ & 0,88 \\
\hline $\begin{array}{l}\text { Tipo de analgesia (número; } \\
\text { sedación/epidural/raquídea) }\end{array}$ & $1 / 20 / 3$ & $1 / 16 / 2$ & \\
\hline Peso recién nacido (grs. media [DE]) & $3363,1(479,7)$ & $3292,5(381,7)$ & 0,56 \\
\hline
\end{tabular}

DE: Desviación estándar;

$n=$ Número de mujeres (en caso de no estar recogida diche variable en todas las participantes). 
Tabla 2. Percepción del dolor

\begin{tabular}{lllll}
\hline & Grupo & $\mathbf{n}$ & $\overline{\mathbf{X}}$ & $\mathbf{D E}$ \\
\hline Percepción de la intensidad del dolor a & Experimental & 25 & 6.99 & 2.68 \\
los $4 \mathrm{~cm}$ de dilatación & Control & 19 & 8.25 & 0,99 \\
\hline Diferencia de medias=-1,26; $I C_{95}$ de la diferencia=-2,56-0,05; $t=-2,16 ; g l=31,95 ; p=0,039$ & & \\
\hline Percepción de la intensidad del dolor & Experimental & 29 & 6,19 & 1,88 \\
global en el puerperio inmediato & Control & 21 & 7,68 & 1,32 \\
\hline Diferencia de medias=-1,48; $I C_{95}$ de la diferencia=-2,40-0,58;t=-3,11; $g l=48 ; p=0,003$ & & \\
\hline
\end{tabular}

Figura 1. Percepción de la intensidad del dolor en el grupo experimental antes de usar la PdP y tras $\mathbf{2 0}$ min. de uso.

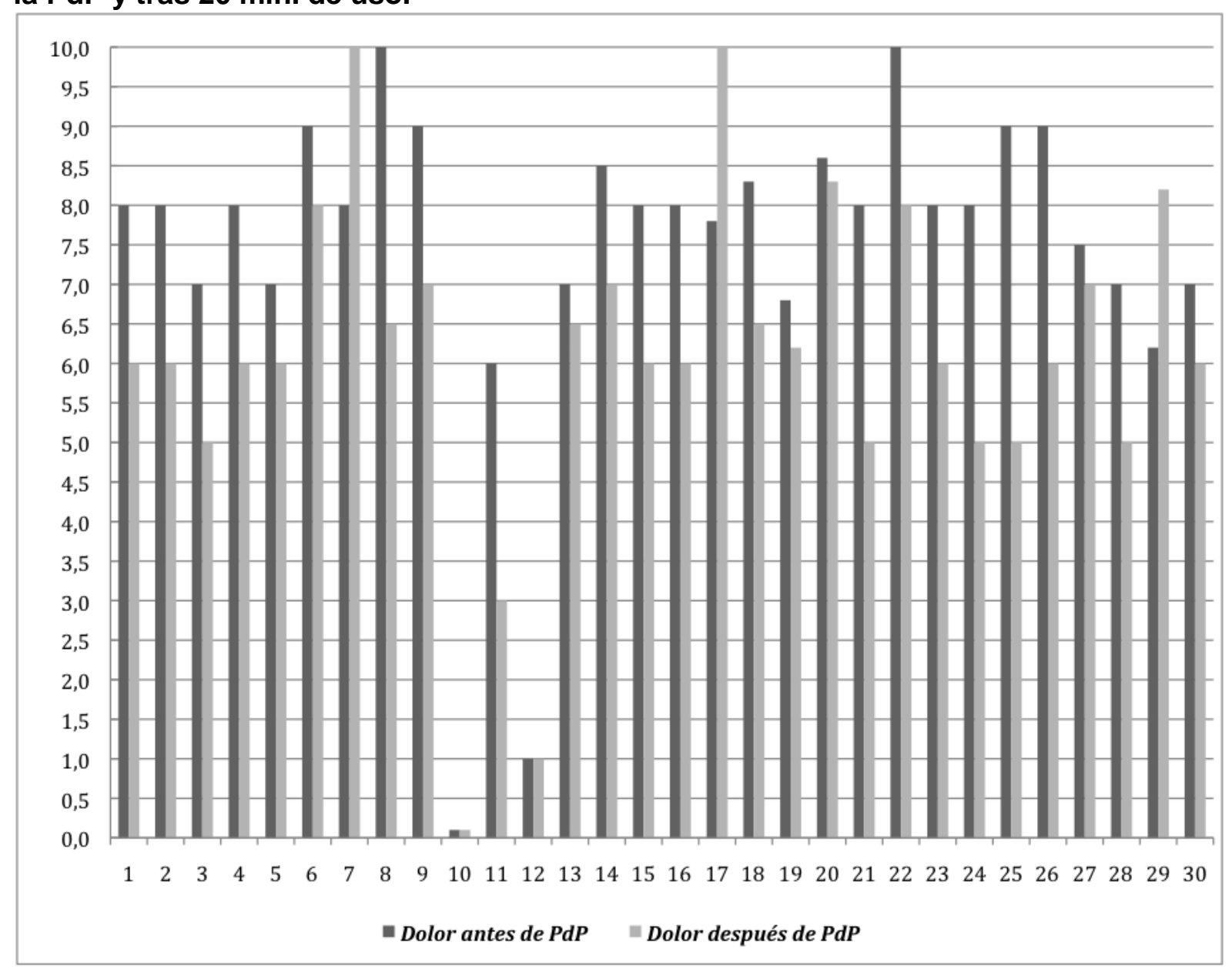


Figura 2. Integridad Perineal

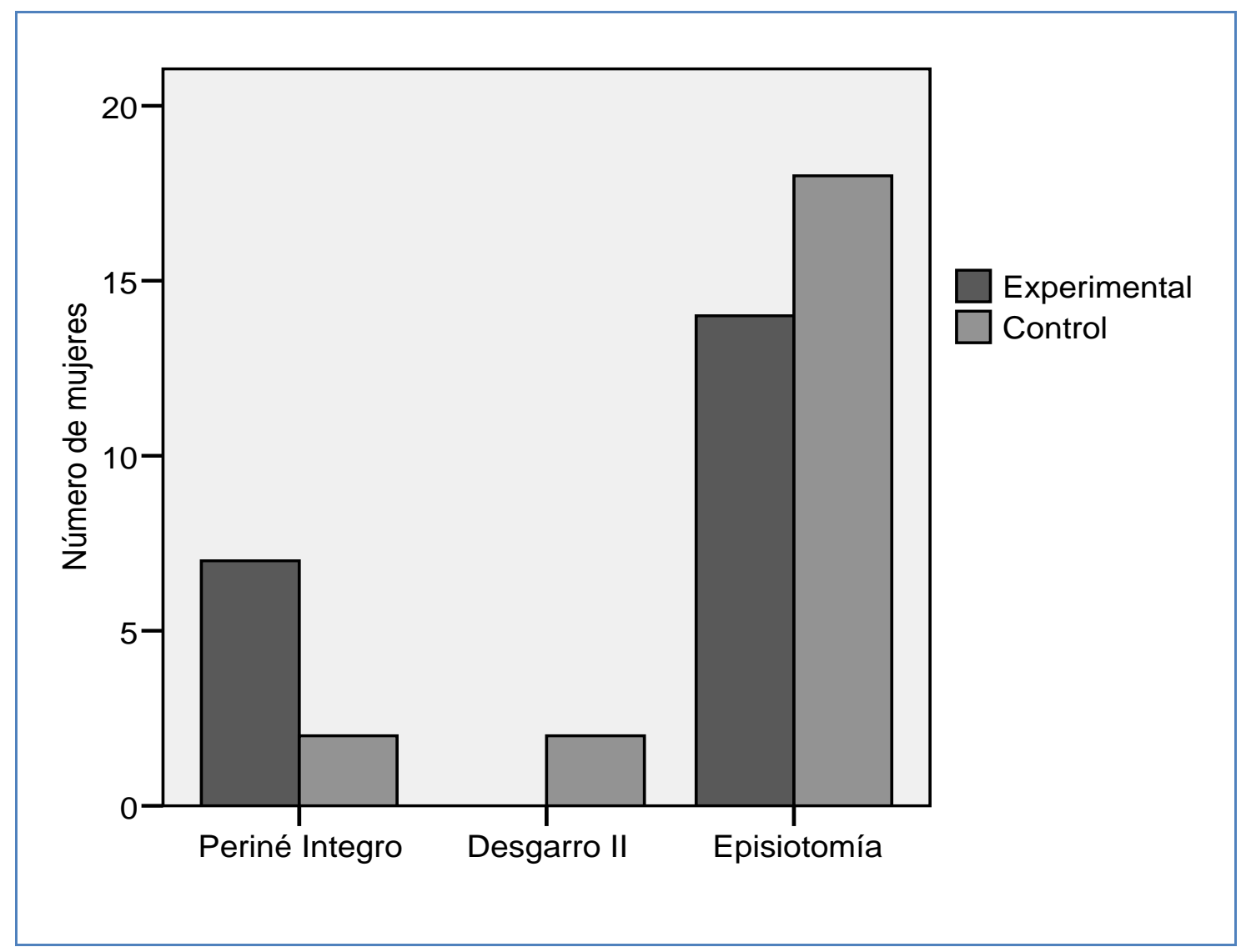




\section{REFERENCIAS}

1. Ministerio de Sanidad y Consumo. Estrategia de Atención al Parto Normal en el Sistema Nacional de Salud, Nov-2007. Madrid (España). (Aprobado por el Consejo Interterritorial el 10 de Octubre 2007). Disponible en URL:

http://www.msc.es/organizacion/sns/planCalidadSNS/pdf/equidad/estrategiaPartoEnero2 008.pdf.

2. Morris SN, Jonson NR. Exercise during pregnancy: a critical appraisal of the literature. J Reprod Med. 2005 Mar; 50 (3): 181-8.

3. Salvesen KA, Morkved S. Randomised controlled trial of pelvic floor muscle training during pregnancy. BMJ 2004 Aug 14; 329 (7462): 378-80.

4. Beckmann MM, Garrett AJ. Antenatal perineal massage for reducing perineal trauma. Cochrane Database of Systematic Reviews 2006, Issue 1. Art. No.: CD005123.

5. Gupta JK, Hofmeyr GJ. Posición de la mujer durante el período expulsivo del trabajo de parto (Revisión Cochrane traducida). En: La Biblioteca Cochrane Plus, 2007 Número 3. Oxford: Update Software Ltd. Disponible en: http://www.update-software.com. (Traducida de The Cochrane Library, 2007 Issue 3. Chichester, UK: John Wiley \& Sons, Ltd.) CD002006.

6. Moral Moreno L. El Fitball una forma diferente y divertida para mejorar nuestra salud. [monografía en Internet]. Disponible en Ball Dynamic's FITBALL®WebSite [Consultado el 15 de Octubre 2007 y el 15 de Enero 2009]

7. Shallow $\mathrm{H}$. My rolling programme. The birth ball: ten years experience of using the physiotherapy ball for labouring women. Midwifery Digest, vol 13, no 1, Mar 2003, pp 2830.

8. Mc Cartey PR. The birth ball_ Are you using it in your practice setting? MCN Am J Matern Child Nurs 1998 Jul-Aug; 23 (4):218.

9. Chang C, Gau M. Experiences applying a birth ball to help a parturient woman in labor. Journal of Nursing [serial on the Internet]. (2006, June), [cited January 20, 2009]; 53(3): 98-103 
10. Wickham S. The risk-managed birth ball. Pract Midwife.2003 Apr 6 (4):35.

11. Anderson T. Me and my birth ball. Pract Midwife. 1998 Sep 1 (9):38.

12. Smith CA, Collins CT, Cyna AM, Crowther CA. Tratamientos complementarios y alternativos para el manejo del dolor durante el trabajo de parto (Revisión Cochrane traducida). En: La Biblioteca Cochrane Plus, 2007 Número 3. Oxford: Update Software Ltd. Disponible en: http://www.update-software.com. (Traducida de The Cochrane Library, 2007 Issue 3. Chichester, UK: John Wiley \& Sons, Ltd.)

13. Hodnett ED. Pain and women's satisfaction with the experience of childbirth: a systematic review. Am J Obstet Gynecol. 2002; 186: S160-172.

14. Stamp G, Kruzins G, Crowther C. Perineal massage in labour and prevention of perineal trauma: randomised controlled trial. BMJ 2001;322:1277-80. 